\title{
Claves para entender el conflicto de Tigray
}

Santiago Molfino*

A un año del inicio del conflicto armado en la región septentrional de Tigray en Etiopía, entre el gobierno federal etíope de Abiy Ahmed Ali y las fuerzas armadas del Frente Popular de Liberación de Tigray (TPLF) - Fuerzas de Defensa de Tigray (FDT)- la situación no ha tendido más que a degradarse y a expandirse de manera inquietante. El grado de violencia perpetrado, la intransigencia de las partes a una salida pacífica y la irrupción de disputas locales paralelas, le imprimen un carácter polifacético de cierta complejidad a la guerra en curso en Etiopía, país central en el Cuerno de África.

El enfrentamiento bélico ha atravesado diferentes fases desde sus comienzos, de repliegue y avances de las diferentes fuerzas en el terreno, lo que ha dado lugar a elucubraciones estratégicas prematuras, a presunciones erradas y subestimaciones que inevitablemente tuvieron su costo táctico, político y militar. En el reverso de estos vaivenes, la violencia generalizada embate de manera implacable a la población, cobrándose miles de vidas. El alarmante escenario humanitario que se vive en la región de Tigray, señala la dimensión crítica actual de una guerra civil que no parece torcer su rumbo. No solo se ha extendido el conflicto en tiempo y espacio, sino que se han incorporado actores regionales y subregionales, que, a través de alianzas fortuitas y motivados por el debilitamiento del gobierno central, ejercen sus demandas propias a partir de un impredecible reordenamiento de fuerzas. Por ello, resulta necesario realizar un breve análisis desde diferentes aristas del conflicto para lograr una comprensión más idónea de lo que está ocurriendo en Etiopía.

\section{Los hechos}

La elección de Abiy Ahmed Ali en abril de 2018 como primer ministro de Etiopía - tras la dimisión de Hailemariam Desalegn en una coyuntura de emergencia y violentas protestas antigubernamentales concentradas en la región de Oromía - marcó el comienzo de una era reformista, de liberalización económica y apertura política anunciada por Ahmed para Etiopía. Desde un inicio, el nuevo proceso se dirigió a desplazar al TPLF del liderazgo central de la coalición gobernante desde 1991, el Frente Democrático Revolucionario del Pueblo Etíope (FDRPE). Ahmed, primer Oromo en conducir la política nacional, concretó la apertura política con la fundación del Partido de la Prosperidad (PP) en 2019, fusionando la antigua coalición con nuevos partidos y apoyándose en alianzas con las élites Oromo y Amhara, las dos etnias mayoritarias del país.

\footnotetext{
" Licenciado en Relaciones Internacionales, Universidad Nacional de Rosario (UNR, Argentina). Miembro del Programa de Estudios América Latina - África (PEALA) del Programa sobre Relaciones y Cooperación Sur-Sur (PRECSUR). E-mail: santiagomolfino@gmail.com
} 
La apuesta por la denominada democracia multipartidista - promesa de campaña de Ahmed bajo su filosofía política Medemer ${ }^{1}$ - se vio afectada desde un principio por el rechazo del TPLF de formar parte de esta, disconforme con el apartamiento de sus principales referentes en la alta cúpula de seguridad del Estado, y en cierta manera, interpretando la pérdida de capital político y los privilegios anexos, como un agravio deliberado de la nueva dirigencia. Aquí se puede observar como la búsqueda por consolidar un proyecto pan-etíope encontró natural resistencia con el modelo de federalismo étnico que el FDRPE - y la elite tigriña como principales artífices - constituyeron como base de gobernanza y construcción de poder por más de dos décadas, logrando cristalizarlo en la constitución etíope de 1993. Este modelo implicó desplazar el peso de la política de la esfera estatal a la regional, una suerte de fragmentación política sostenida en un rediseño administrativo del país que permitió que el TPLF representando una etnia de poco peso demográfico y confinada al extremo norte del país - se consolidase estratégicamente en el poder y evitase el surgimiento de una oposición nacional de peso. La irrupción de Ahmed, en ese sentido, constituyó un cimbronazo político.

Entonces, esta incompatibilidad ideológica y práctica, preanunció de alguna manera un conflicto que nunca encontró un canal formal de resolución. El repliegue del TPLF a la gobernanza de su región, confirmó el distanciamiento cada vez más marcado con el gobierno federal. El desacato del TPLF a éste último, de llevar las elecciones regionales de agosto de 2020 - postergadas por la pandemia Covid-19 - constituyó el comienzo de un conflicto que escaló de manera acelerada y agresiva. La respuesta de Addis Abeba, congelando la transferencia de fondos presupuestarios a Tigray, declarando la inconstitucionalidad de los comicios y la ilegalidad del nuevo gabinete legislativo de Tigray, recibió respuesta inmediata del TPLF que manifestó que no reconocería a la administración de Abiy después de que expirara su mandato original el 5 de octubre de 2020. Esto implicó el respaldo casi unánime del electorado local tigriño a la decisión del partido de desafiar a las autoridades federales, lo que configuró el corrimiento de una disputa constitucional en un marco democrático hacia una declaración de guerra. Finalmente, las FDT atacaron una base militar nacional del oeste de la región y secuestraron artillería pesada, lo que provocó la intervención militar inmediata por parte de las Fuerza de Defensa Nacional Etíope (FDNE) "para salvar el país y la región"².

La ofensiva concentrada del gobierno de Ahmed que denominó "aplicación de ley limitada" 3 -que incluso lo llevó a oficializar la victoria federal con la toma de Mekelle a finales de noviembre de 2020 - denota hoy una presunción errada, y la subestimación del poderío de tropas del TPLF, que más allá del revés inicial, pudo diseminar una resistencia acendrada en la región. Por su parte, Debretsion Gebremichael, líder del TPLF, emitía afirmaciones de "victorias en el campo de batalla de las Fuerzas de Defensa de Tigray"4, aún semanas después de su pérdida del control del gobierno regional y del anuncio por parte de Addis Abeba de que la guerra había entrado en su "fase final". La recuperación de la capital tigriña por parte de las FDT en junio de este año - en la cual se vieron marchando cientos de soldados federales prisioneros- permiten señalan la aleatoriedad de los hechos, y la pervivencia de una disputa, que aún en sus fases de baja intensidad, nunca permitió el fortalecimiento total de la toma de posiciones.

\footnotetext{
1 Medemer se traduce como sinergia, estar incluido o estar juntos. Abiy propone este principio de filosofía política como guía para desarrollar un entendimiento compartido en amplios temas como la historia, la cultura y una forma de construir una nueva administración política. La noción es muy relevante en esta coyuntura del desarrollo político de Etiopía, ya que afirma proporcionar la salvación de la profundización de las divisiones etnolingüísticas y avanzar hacia la conformación de una Gran Etiopía.

2 Ver en https://www.aljazeera.com/news/2020/11/4/pm-ahmed-vows-response-after-deadly-attack-innorthern-ethio pia

${ }^{3}$ Ver en https://addiszeybe.com/news/currentaffairs/law-enforcement-operation-or-an-armed-conflict-theconflict-in-tigray-and-international-humanitarian-law

4 Ver en "Informe del Gobierno Regional Nacional de Tigray sobre el actual conflicto en Etiopía", Oficina de Enlace de Amistad de Tigray, 2 de diciembre de 2020.
} 
Desde el comienzo, el gobierno central buscó aplacar al TPLF, a través de la persecución feroz de sus líderes principales - esto incluyó a ex líderes políticos y militares del período hegemónico del Frente Democrático Revolucionario del Pueblo Etíope (EPRDF) - con el objetivo directo de asesinato o arresto, generar una rendición automática de las tropas enemigas y desmontar su aparato político y red de vínculos centrales. Por ello, en enero de 2021, el gobierno federal despojó al TPLF de su condición de partido legal y en mayo, le aplicó la designación legal de organización terrorista por "liderar actividades destructivas contra la nación"

En el plano político legal, Etiopía celebró el último 22 de junio las elecciones generales y regionales postergadas sucesivamente por Covid-19, lo que constituyó otro hecho a subrayar en este año de hostilidades. Esta instancia fue la primera prueba electoral afrontada por el premier Abiy Ahmed desde su llegada al poder en 2018 y a través de la cual logró revalidar el cargo. De 547 distritos electorales, 110 se vieron privados de votar por la creciente inseguridad en el país. Esto se comprobó con la reducida oferta electoral a causa de la exclusión del TPLF del proceso, así como con el retiro de los principales partidos de oposición -Frente de Liberación Oromo (OLF) y el Congreso Federalista Oromo (OFC)- debido al encarcelamiento de algunos de sus líderes y el cierre de sus oficinas por manos del gobierno. Como corolario, el gobierno del PP extendió su administración por un lustro y su voluntad política será crucial para una negociación con el TPLF en el corto y mediano plazo. Tan volátil resulta el panorama, que una semana después de las elecciones Ahmed - en un clima de afianzamiento democrático de apoyo a su figura y al manejo del conflicto- llamó a un "alto al fuego unilateral" 6 a raíz de la ya mencionada recuperación de Mekele por parte de las fuerzas de Tigray, acaecida la misma semana.

Ahora bien, cabe señalar que la medida de excepción de Estado de emergencia - el segundo en lo que va del conflicto y de graves antecedentes recientes en Etiopía - declarado oficialmente por Ahmed a principios de noviembre del corriente (aprobado por el Parlamento Nacional) luego de que las FDT avanzaran hacia la vecina región de Amhara, convalida una recusable persecución étnica, no ya a líderes sino a la población tigré presente en la capital del país. Esta modalidad de detención de la población sin orden judicial bajo "sospecha razonable" de cooperación con "grupos terroristas" -- viola el derecho Internacional de los Derechos Humanos, que prohíbe la detención arbitraria y la discriminación en todas las circunstancias. ${ }^{7}$ Además de esta medida, el gobierno llevó a cabo un reclutamiento masivo de jóvenes para engrosar las tropas federales, en una campaña oficialista sin precedentes donde Ahmed ungió a los ciudadanos a "dejar temporalmente sus asuntos cotidianos en espera y a unirse y a organizarse para poner todas las armas y recursos al servicio de la guerra contra el TPLF" ${ }^{8}$.

\section{Batalla narrativa y asedio}

El corte total de servicios de telecomunicaciones ha sido otro determinante del conflicto. La inaccesibilidad a la información y a los reportes de medios de comunicación - a raíz del blackout total establecido por el gobierno federal en el Tigray- ha llevado a que ambas partes se incriminen mutuamente respecto a crímenes cometidos en la región, se contradigan respecto a maniobras en el territorio e intenten sustraer a la opinión pública local - e internacional- del verdadero curso de los hechos. Las redes sociales también se convirtieron en un campo de batalla, con el gobierno etíope y sus partidarios por un lado y los activistas y partidarios del TPLF por el otro, donde cada uno intentó presentar su propia versión de los

\footnotetext{
${ }^{5}$ Ver en https://www.aljazeera.com/news/2021/5/1/ethiopia-to-designate-tplf-olf-shene-as-terror-groups

${ }^{6}$ Ver en: https://www.france24.com/es/\%C3\%A1 frica/20210628-etiopia-gobierno-abiy-ahmed-altofuego-tigray

${ }^{7}$ Ver en https://www.amnesty.org/es/latest/press-release/2021/03/ethiopia-un-human-rights-chiefunderscores-urgency-of-impartial-international-investigation-into-tigray-atrocities/

${ }^{8}$ Ver en: https://www.dw.com/es/etiop\%C3\%ADa-gobierno-declara-estado-de-emergencia-en-todo-el-pa \%C3\%ADs/a-59699363
} 
sucesos. Si la comunicación del TPLF se centró en crear conciencia sobre el conflicto, los partidarios de la administración del primer ministro Abiy Ahmed en Addis Abeba intentaron refutar las afirmaciones de su oponente. Sin embargo, las comunicaciones oficiales y las publicaciones de los usuarios progubernamentales buscaron desacreditar cualquier contenido que contradijera la narrativa del gobierno federal como desinformación. En adición, el estado etíope juega un papel monopólico en el panorama de los medios en el país, siendo dueño directo de al menos un tercio de todos los medios de difusión ${ }^{9}$, lo que sugiere una disparidad marcada.

Esto ha insuflado aún más la línea discursiva nacionalista de Addis Abeba, que ha instrumentalizado el enfrentamiento con el enemigo interno como causa nacional de supervivencia. La reciente avanzada de las tropas de Tigray hacia el sur tomando las ciudades de Kombolocha y Kemise, como puntos estratégicos rumbo a Addis Abeba -situación que el gobierno federal ha calificado como un "período turbulento" a superar con "resiliencia nacional" - refleja el discurso centralista de la Gran Etiopía. Sin lugar a segundas interpretaciones, el propio Ahmed sentenció: "Sacrificaremos nuestra sangre y nuestros huesos para enterrar al enemigo y defender la dignidad y la bandera de Etiopía"10.

En definitiva, la génesis de una disputa de dos modelos ideológicos y políticos encuentra en este contexto su perfecta puesta en escena. El vacío de información sobre la guerra, y la disputa diaria por la comprensión del conflicto deben analizarse en esta dirección. El ocultamiento ante la comunidad internacional de crímenes cometidos por todos los actores implicados, la alta producción de fake news y el efecto del sesgo mediático sobre una población partida y atemorizada no abonan a ningún tipo de salida conciliatoria. En efecto, la batalla por la narrativa - manipulando plataformas comunicacionales digitales y propaganda- ha sido por momentos de mayor preocupación por los contendientes que el impacto real de la guerra. Es decir, el conflicto físico y el informativo se alimentan deliberadamente entre sí, donde se busca perpetuar una narrativa particular, y aún más peligroso, interpone la cuestión étnica -se presenta como un asunto existencial que les concierne a las etnias enfrentadas - como eje de la disputa, ante las reiteradas denuncias de limpieza étnica o crímenes que circundan el genocidio.

El hermetismo informativo imperante ha sido utilizado por el gobierno federal para sellar el asedio al que se encuentra sometido la región de Tigray, fundamentalmente a través del impedimento de acceso a la asistencia humanitaria ${ }^{11}$, como ha sido denunciado en reiteradas ocasiones por organizaciones internacionales y agencias humanitarias que mantiene actividad diaria en el terreno. La Organización de Asuntos Humanitarios (OCHA) manifestó que unos 5,2 millones de personas en Tigray, más del 90\% de la población de esa región etíope, precisan asistencia de emergencia para sobrevivir, y que actualmente ya son unas 400.000 personas las que enfrentan condiciones de hambruna (IPC ${ }^{12} 5$ : nivel 'catastrófico' de inseguridad alimentaria) y más de 4 millones de personas - 70\% de la población - experimenta altos niveles de inseguridad alimentaria aguda (IPC 3 o superior).

La utilización de la hambruna como arma de guerra demuestra la gravedad de la situación, más aún con la destrucción de aldeas rurales que ha impedido que los agricultores y ganaderos puedan cosechar provocando escasez en los mercados. Antes de la escalada bélica, Tigray era una de las regiones agrarias más ricas de la nación, es por esto que los ciclos de cultivo, lluvia y cosecha constituyen factores centrales que marcan el manejo de los tiempos del conflicto y las maniobras del gobierno central. El Programa Mundial de Alimentos (PMA)

\footnotetext{
${ }^{9}$ Para ampliar, ver: "Fake News Misinformation and Hate Speech in Ethiopia: A Vulnerability Assessment", Instituto Europeo de la Paz, pág.6, pág.9, 12 de abril de 2021, https://www.eip.org/wpcontent/uploads/2021/04/Fake-News-Misinformation-and-Hate-Speech-in-Ethiopia.pdf

10 Ver en: https://mobile.twitter.com/Reuters/status/1456037221975277571.Discurso Abiy Ahmed $\underline{3 / 11 / 2021}$

${ }^{11}$ Human Rights Watch (HRW) por su parte, advirtió que "las acciones que impidan deliberadamente los suministros de socorro" violan el derecho internacional humanitario

${ }^{12}$ The Integrated Food Security Phase Classification (IPC) es una iniciativa innovadora de múltiples socios (ONU; ONGs y otros actores de la sociedad civil) para mejorar el análisis y la toma de decisiones de la seguridad alimentaria y la nutrición.
} 
sostiene que se requerirá asistencia alimentaria al menos hasta la temporada de cosecha del próximo año durante el último trimestre de 2022, ya que la temporada de siembra agrícola se perdió por completo en gran parte de la región.

Por otra parte, el control de las rutas de suministros también constituye un eje importante en la estrategia del TPLF. Dicho esto, el TPLF y el Ejército de Liberación Oromo (OLA) - aliado principal tigriño - concentran los esfuerzos en avanzar hacia la región centro oriental de Afar, para consolidar un mayor control sobre las rutas estratégicas que unen Etiopía - país mediterráneo - con Yibuti, principal arteria comercial para la salida marítima de las exportaciones etíopes. De concretarse, asestaría un golpe importante al gobierno central. Yibuti - enclave sede de las principales bases militares extranjeras entre ellas las de China, Estados Unidos, Italia, Francia y Japón- ha militarizado la frontera ante una posible avanzada de las fuerzas mencionadas, lo que podría significar un rol activo de las potencias en el conflicto.

\section{Multiplicidad de actores, alianzas y riesgo regional}

Etiopía vive su crisis de seguridad más severa en décadas, y esto se debe principalmente, a la expansión del conflicto en múltiples frentes y la emergencia de nuevos -y viejos- actores internos y externos que intentan sacar provecho de la inestable correlación de fuerzas a través de la lucha armada. Se observa así una superposición de conflictos entre milicias regionales que responden a la dinámica de la contienda central. El debilitamiento del gobierno federal por la apertura de múltiples frentes y la imposibilidad de cobertura de las tropas federales expone su poder de acción y limitaciones, lo que paulatinamente amplifica otros focos calientes en zonas y woredas $^{13}$ de las diferentes regiones administrativas aledañas del Tigray.

La temprana intrusión - a efecto inmediato del bombardeo confirmado del TPLF al aeropuerto de la capital Asmara el 15 de noviembre de 2020- de Eritrea a través de las Fuerzas Eritreas de Defensa (EDF) en la guerra señaló la siempre posibilidad latente de que el conflicto se derrame por las fronteras ya inflamadas del Cuerno de África. La relación entre Eritrea y Etiopía es posiblemente una de las principales fuentes de inestabilidad regional en esta región, de allí la trascendencia que tuvo el nuevo período de paz propulsado por la administración de Ahmed - luego del estancamiento de 20 años posterior a la guerra fronteriza que mantuvieron entre 1998 y 2000- para reequilibrar lazos regionales y consolidar a Etiopía como país rector del Cuerno.

La participación eritrea en el conflicto, en este sentido, implica un claro retroceso para la desmilitarización regional y peor aún, contemplando que el TPLF constituye para el régimen de Isaías Afewerki una amenaza existencial desde hace años y la posibilidad de que se instale una guerra de baja intensidad y obstaculice una hipotética voluntad de negociar por parte de Addis Abeba. Resulta inquietante, dada la actualidad del panorama. preguntarnos si no fue la reconciliación histórica entre ambos países un prefacio para suprimir al TPLF de manera definitiva, hoy adversario común de ambos líderes. La visita de Afewerki en octubre de 2020 a los emplazamientos militares etíopes - en la antesala del estallido bélico - debe entonces interpretarse en ese sentido.

Después de meses de negar de manera sistemática ante la comunidad internacional su presencia en territorio etíope, el gobierno de Eritrea finalmente justificó ${ }^{14}$ la invasión de Eritrea como una "medida legítima" de autodefensa contra la "amenaza regional" que representa el TPLF. Si bien, tanto los gobiernos de Eritrea y Etiopia se comprometieron al retiro de tropas extranjeras, en los hechos, las EDF continúan en ejercicio no solo en la región de Tigray, sino

\footnotetext{
${ }^{13}$ Nombre que reciben las circunscripciones administrativas, o gobiernos locales, en que se dividen las regiones de Etiopía; son equivalentes a un distrito.

${ }^{14}$ Esta declaración oficial fue a través de una carta enviada a la ONU por la embajadora eritrea, Sophia Tesfamariam. Ver en https://africatimes.com/2021/04/17/eritrea-confirms-military-presence-in-ethiopiain-un-security-council-letter/ Consultado el 22/06/2021.
} 
también en la contigua región de Amhara. Esta presencia podría volverse permanente si las Fuerzas de Defensa nacional de Etiopía (ENDF) continúan exigidas - lo cual implicaría no poder prescindir del apoyo eritreo- y Afewerki decide avanzar con su cruzada contra el TPLF. Si un período de transición liderado por este último ocurriese, no se podría descartar que el gobierno de Eritrea probablemente apoye a grupos insurgentes - a través de guerras subsidiarias o indirectas (proxy war) dispersas en la región - como ocurrió durante casi dos décadas en que la elite tigriña gobernó Etiopía a través del EPRDFE.

En el escenario nacional, la ramificación del conflicto en tramas y subtramas responde esencialmente a la composición multiétnica de Etiopía, que presenta más de 90 grupos étnicos diferentes y una infinidad de lenguas vivas presentes en el territorio. La división reordenamiento administrativo en regiones - con selecta representación de etnias mayoritarias en regiones asignadas bajo un criterio etnolingüístico- establecido por el EPRDFE en la constitución de 1993 constituye hoy, en retrospectiva, quizás la piedra angular de las tensiones intracomunitarias. Los desplazamientos forzados que privan la libre movilidad por el territorio, la falta de acceso a la tierra por parte de etnias minoritarias y la imposibilidad de ejercicio del derecho civil y político - y representación política- de éstas configuran alguna de las cuestiones que fueron omitidas deliberadamente por los sucesivos gobiernos centrales de Etiopía. La omisión de buscar solución a estos puntos sensibles de fricción demuestra la inefectividad del federalismo étnico como sistema político, o al menos, las ambigüedades que presenta.

La participación activa de las milicias y fuerzas especiales de Amhara - segunda etnia mayoritaria del país- se ubican como el principal aliado de las fuerzas federales en la guerra civil en curso. La ocupación amhara de los territorios de Gichew y Gobe de Tigray - que el gobierno regional de Amhara considera arrebatadas y anexadas unilateralmente en los años noventa por la dirigencia Tigré - vino acompañado de fuerte presencia militar en el terreno, severos crímenes y la expulsión de miles de habitantes de dicha región hacia la frontera con Sudán.

Otro caso a señalar es el del OLA, que con los últimos acontecimientos y el avance del TPLF hacia la capital, constituye la alianza - junto a siete grupos armados insurgentes ${ }^{15}$ cuyo objetivo es reemplazar el gobierno de Ahmed - más importante a tener en cuenta. Enfrentados en el pasado, en la actualidad aliados, no resulta claro si el TPLF y la OLA intentarán tomar el control de Addis Abeba o efectivamente buscan presionar al gobierno federal para que ceda en concesiones sustanciales, pero dado que las negociaciones políticas parecen lejanas, el conflicto se orienta en esa dirección mientras el TPLF busca romper el asedio de Tigray en el campo de batalla.

El Frente de Liberación Oromo (FLO) - organización independentista etíope que lucha por la autodeterminación del pueblo oromo - y su brazo armado, el OLA, han sido denunciados por la dirigencia Amhara por haber librado una "guerra relámpago" en los últimos meses signada por "masacres de civiles y destrucción masiva" en territorio Amhara ${ }^{16}$. La región de Oromía - estado natal de Abiy - se ha visto convulsionada con protestas incluso antes de la disputa militar, ante el reclamo de que el primer ministro incumplió sus promesas y no avanzó hacia la concreción de las demandas postergadas de dicha región, en beneficio de otorgarle más centralidad a los intereses de la élite amhara, cuyo apoyo resulta clave para el delicado equilibrio político en que se encuentra el gobierno central. Como resultado de esta carrera para influir sobre las decisiones del gobierno central, las rupturas y pérdidas de apoyo implican una frágil situación al interior del PP, condicionado por la exigencia de una actitud más agresiva por parte de la dirigencia amhara para que contenga la avanzada del OLA y el TPLF en el terreno.

\footnotetext{
${ }^{15}$ Los firmantes de la alianza fueron: Afar Revolutionary Democratic Unity Front; Agaw Democratic Movement; Benishangul People's Liberation Movement; Gambella Peoples Liberation Army; Global Kimant People Right and Justice Movement/ Kimant Democratic Party; Sidama National Liberation Front; Somali State Resistance. Ver en: https://www.africanews.com/2021/11/05/ethiopia-9-rebel-groupsjoin-forces-against-the-government/

16 Ver en https://www.theafricareport.com/84350/ethiopias-war-in-tigray-is-but-the-tip-of-the-icebergwhen-it-comes-to-conflicts-ravaging-the-country/
} 
La frontera occidental con Sudán es otro riesgo de spill over regional, ya que el ejército sudanés también ha tomado parte activa en el conflicto. La disputa fronteriza entre Sudán y Etiopía que comenzó en noviembre de 2020 sobre la zona denominada al-Fashaga -área ubicada entre la provincia oriental sudanesa de Al Qadarif y los estados regionales de Amhara y Tigray de Etiopía - registra un recrudecimiento. Pese al acuerdo de 2007 bajo los oficios de Meles Zenawi - mediante el cual se permitió la libre movilidad de agricultores de un lado y otro de la frontera y la posibilidad de comerciar en territorio etíope- las Fuerzas Armadas Sudanesas (FAS) han ocupado el territorio en mención, militarizando la frontera y asentándose incluso con la rauda construcción de infraestructura (carreteras, puentes, bases de operaciones de avanzada) y expulsando a paramilitares etíopes de la zona, aprovechando el vacío de seguridad creado por el enfrentamiento armado del gobierno federal con el TPLF. Esta consolidación del control sudanés probablemente busque asegurar beneficios que sean más difíciles de revertir a futuro. Addis Abeba, por su parte, acusó a Sudán de invadir su territorio y respondió a estos acontecimientos enviando refuerzos acompañados por milicias amhara al territorio en disputa.

En la tercera semana de diciembre de 2020, las tropas etíopes y sudanesas se enfrentaron en combate directo por primera vez en un cuarto de siglo. Advirtiendo que la ruta de suministro clave de las FDT para romper su asedio es Sudán - como lo fue durante la década de 1980 contra la dictadura militar de Mengistu Haile Mariam - una potencial guerra con Sudán podría ofrecer a la resistencia de Tigray una oportunidad de extender su influencia más allá de las áreas rurales en el centro y sur de Tigray que controla. Esto también podría reforzar la dependencia del gobierno etíope de las fuerzas militares de Amhara y de Eritrea para contener este avance, pero fundamentalmente se presenta como una seria amenaza para la estabilidad de ambas naciones - ya dañada con el golpe de estado militar sudanés del 25 de octubre del corriente y su trunca transición hacia un gobierno civil- que podría impactar de manera sumamente perniciosa en el Cuerno de África.

Pese a las hostilidades limítrofes, la frontera sudanesa continúa siendo la principal ruta de escape de la población civil que busca asilo. Según la Agencia para Refugiado de Naciones Unidas (UNHCR) desde que inició el conflicto, 2,1 millones de desplazados internos (PDI) estimados en la región de Tigray, también hay 250,000 PDI en la región de Amhara y 112,000 en la región de Afar. No obstante, se estima que más de 50.000 refugiados etíopes provenientes de las regiones de Tigray, Benishangul-Gumuz y Amhara han huido a territorio sudanés ${ }^{17}$.

La Gran Presa del Renacimiento Etíope (GERD) - a sólo $15 \mathrm{~km}$ de la frontera sudanesa- que Etiopía procedió a su segundo llenado, es otro foco caliente de suma relevancia que involucra a ambos países e inflama la región. La fuerte impronta militarista que impera podría llevar a una propaganda belicosa en ascenso con respecto a su frontera y a la desestimación de llegar a un acuerdo a través de la mediación de la Unión Africana. Ésta intentará por segunda vez - esta vez bajo los oficios de la República Democrática del Congo lograr un marco formal de negociación, luego de que el Consejo de Seguridad de ONU devolviese el asunto a la égida de la Unión Africana (UA). Un dato a destacar, es la firma en junio de este año de un acuerdo de cooperación militar - respaldados por la Liga de los Estados Árabes- entre Sudán y Egipto, el otro actor de peso enfrentado a Etiopía por su gestión unilateral del cauce del Nilo, de cuyas aguas depende casi en su totalidad y considera un factor existencial para su desarrollo (ante una amenaza al suministro de agua).

\section{Mucho en juego, pocas respuestas}

Etiopía conjuga mucho de los intereses de los key players globales en el tablero geopolítico del Cuerno de África. Ante el escenario de conflagración, encontramos posturas disímiles pero todas exiguas. China, por un lado, factor dinamizador principal en el ascenso etíope en el siglo XXI, se mostró reacia a desempeñar un papel más destacado en los intentos de resolver el conflicto de Tigray, en parte debido a su política de no injerencia en los asuntos

\footnotetext{
${ }^{17}$ Ver en: https://www.unrefugees.org/news/how-unhcr-is-helping-ethiopian-refugees-fleeing-to-sudan/
} 
internos de otros países; siempre manteniendo un perfil bajo con motivo de pisar con cuidado en un país estratégico para sus proyecciones geopolíticas en el marco de la iniciativa de la Franja y la Ruta. Rusia -que rubricó un acuerdo en 2018 con Etiopía de apoyo en varios sectores incluyendo el apoyo técnico a sus fuerzas armadas - también ha adoptado un enfoque similar al de China hacia la guerra de Tigray. Si bien tuvo un papel clave en el bloqueo de una declaración unánime de la ONU sobre la violencia en Tigray, considera que tal resolución violaría su compromiso de no interferencia en los asuntos internos de los estados. Esto explica en parte la pasividad del Consejo de Seguridad (CS) ante la expulsión por parte del Gobierno de Etiopía de siete altos funcionarios de agencias humanitarias de la ONU que fueron declarados "persona non grata" por "entrometerse en los asuntos internos del país"18.

Si bien la nueva administración demócrata en Estados Unidos prometió "la vuelta del multilateralismo", la guerra de Tigray en Etiopía continúa llevando a los límites de los mecanismos humanitarios de la ONU y exhibe la falta de respuestas ante el bloqueo en el CS. Las últimas - y únicas- medidas que Estados Unidos tomó hasta el momento fueron la suspensión de las preferencias arancelarias --que otorga una ley estadounidense a países del África subsahariana (AGOA) - a partir de enero de 2022, el congelamiento de activos en el exterior y la suspensión de visas a funcionarios etíopes. A pesar de que la ayuda humanitaria proporcionada por los organismos de ONU continúa siendo vital, el disfuncionamiento de posiciones en el CS impide apuntalar un proceso de mediación liderado por la Unión Africana (UA), mediante asesoramiento especializado y asistencia técnica. Los oradores del Consejo de Seguridad instan al diálogo político entre las partes para prevenir desastres y confirman su apoyo a los esfuerzos de paz de la UA en Etiopía, lo cual, ante la magnitud de la situación humanitaria, resulta un desmarque evidente de la situación en territorio etíope. En definitiva, el CS estuvo paralizado durante meses antes de expresar preocupación por la creciente gravedad de la situación en Tigray, y la situación reciente en Afganistán, solapó el tema de su agenda, donde recién se lo incluyó en el mes de octubre de 2021, para tratarla en sesiones ordinarias.

Etiopía posee una posición preeminente en el Cuerno de África, en el cual se presenta como potencia africana emergente - es el país de mayor potencial económico de la región con acceso a un amplio mercado y una población de más de 115 millones de habitantes, el segundo más populoso de todo el continente- dado el crecimiento sin precedentes manifestado en la última década. A su vez, su rol de estabilizador regional también es serio. Durante el primer tramo de gobierno de Ahmed, se observó un giro político iniciado en el Cuerno de África, potenciado por la política exterior etíope, que buscó ser la pieza central para revitalizar la cooperación regional, tratando de profundizar las ganancias diplomáticas, socioeconómicas y perfilar un esquema de relativa estabilidad, paz y seguridad en el Cuerno de África. Esto tuvo logros varios: la paz con Eritrea; el acuerdo de paz de transición en Sudan de 2019 auspiciado por el propio Ahmed; la reapertura de relaciones diplomáticas entre Yibuti y Eritrea; la sorpresiva alianza tripartita de cooperación y desarrollo entre Etiopía, Somalia y Eritrea.

Sin embargo, el retorno intempestivo a la militarización por parte del gobierno federal reflotó las turbulencias en la región y desmontó la red de vinculaciones interestatales estables construida con tanto esfuerzo. Addis Abeba, epicentro del multilateralismo regional africano sede de la UA y de la Comunidad Económica para África (CEPA) - y de su relacionamiento diplomático con la comunidad internacional adhiere una presión mayor a la búsqueda de un alto al fuego en el conflicto de Tigray. La preocupación ahora es que el conflicto está entrando en una nueva fase y será cada vez más difícil que cualquiera de las partes ceda para llamar a una mesa abierta de negociación, más aún, careciendo la región de un marco de seguridad regional eficiente.

Para el Gobierno de Abiy Ahmed, por ahora negociar con el TPLF no es una opción, y advierte a la ONU de que "respete la ley"19. Desde el inicio del enfrentamiento, el gobierno de

\footnotetext{
${ }^{18}$ Ver en: https://news.un.org/en/story/2021/10/1102352

${ }^{19}$ Ver en https://www.reuters.com/world/africa/ethiopia-says-un-staff-will-face-penalties-if-they-breaklaw-2021-11-11/
} 
Etiopía ha exhortado a la comunidad internacional a que se mantenga fuera de los asuntos internos de su país, rechazando todas las acusaciones de crímenes cometidos en el territorio. En esta última fase, Addis parece reconocer los "esfuerzos" - no fue así con el intento de la Autoridad Intergubernamental sobre el Desarrollo (IGAD)- del Alto Representante de la Unión Africana (UA) para el Cuerno de África, el ex presidente nigeriano Olusegun Obasanjo, con quien ha aceptado abordar el conflicto. Desde otro ángulo, la mediación de Obasanjo también incluye negociar con los líderes TPLF- situación que efectivamente sucedió a inicios de noviembre de este año en Mekele - y otorgarle validez como actor político, lo que el gobierno central etíope considera intolerable dada su calificación de "grupo terrorista". En conclusión, este conflicto se pueda convertir en un catalizador de tensiones dado el peso de Etiopía en el Cuerno, imponiendo tendencias desestabilizadoras en una región que parece resignarse a la vía militar como modus vivendi. Es de suma trascendencia lo que se juega en Etiopía. Si bien resulta apremiante la paz y el alto al fuego humanitario, de concretarse una salida política, deberá conllevar profundos cambios de organización política, reforma constitucional principalmente el derecho de acceso a la tierra - sopesando las cuestiones de la autonomía regional y la autodeterminación; y finalmente abordando las tensiones interétnicas de fondo. De lo contrario, la desintegración nacional etíope dejará de sonar como una letanía.

TRABAJO RECIBIDO: 17/11/2021 